\title{
Dose-Optimization of Respiratory-Gated Auricular Vagal Afferent Nerve Stimulation (RAVANS) for Blood Pressure Modulation in Hypertensive Patients
}

\author{
Jessica Stowell ${ }^{1}$, Ronald G Garcia ${ }^{1,2,3}$, Rachel Staley ${ }^{1}$, Roberta Sclocco ${ }^{2,4}$, Harrison Fisher ${ }^{2}$, \\ Vitaly Napadow ${ }^{2,4}$, Jill Goldstein ${ }^{1,2}$, Riccardo Barbieri ${ }^{5,6}$ \\ ${ }^{1}$ Department of Psychiatry, Massachusetts General Hospital, Harvard Medical School, Boston, USA \\ ${ }^{2}$ Athinoula A. Martinos Center for Biomedical Imaging, Department of Radiology, Massachusetts \\ General Hospital, Harvard Medical School, Boston, USA \\ ${ }^{3}$ School of Medicine, Universidad de Santander (UDES), Bucaramanga, Colombia \\ ${ }^{4}$ Department of Radiology, Logan University, Chesterfield, MO, USA \\ ${ }^{4}$ Department of Electronics, Information and Bioengineering, Politecnico di Milano, Italy \\ ${ }^{5}$ Department of Anesthesia, Critical Care and Pain Medicine, Massachusetts General Hospital, \\ Harvard Medical School, Boston, USA
}

\begin{abstract}
The objective of this study was to determine the optimal frequency of respiratory-gated auricular vagal afferent nerve stimulation (RAVANS) for the modulation of blood pressure in hypertensive patients.

Twelve hypertensive subjects $(52.5 \pm 6.0$ years, 8 females) underwent five randomized stimulation sessions, during which they received exhalatory-gated stimulation at frequencies of 2, 10, 25, and $100 \mathrm{~Hz}$ or sham stimulation. A continuous blood pressure signal was collected during a 30-minute stimulation period and a 10minute recovery period using a Finometer device (Finapress Medical System, the Netherlands). LabChart (ADInstruments, Colorado Springs, CO, USA) was used to process and compute blood pressure responses.

A significantly greater reduction of systolic blood pressure values during stimulation was observed in the $100 \mathrm{~Hz}$ session compared to sham $(p=0.02)$. In addition, significant reductions in diastolic blood pressure $(p=0.04)$ and mean arterial pressure $(p=0.04)$ values were observed during RAVANS stimulation compared to baseline during the $100 \mathrm{~Hz}$ session. Evaluation of other stimulation frequencies did not reveal significant results.

RAVANS exhibits a frequency-dependent effect on the modulation of arterial blood pressure levels of hypertensive subjects.
\end{abstract}

\section{Introduction}

Hypertension affects the health of 1 in 3 Americans and is the direct cause of mortality for more than 410,000 individuals each year [1]. It has been reported that 7 out of
10 adults with hypertension in the United States have been prescribed antihypertensive medications, yet half of those patients remain with uncontrolled blood pressure. Additionally, from 1980 to 2008, the worldwide population of patients with uncontrolled hypertension increased from 605 million to 978 million [1]. Although antihypertensive medications are widely prescribed and utilized, there is still a high demand for a more effective treatment of hypertension across the world.

One etiological mechanism that has been proposed for hypertension is cardiovascular autonomic dysregulation [2]. Vagus nerve stimulation (VNS), an implanted therapeutic for major depression [3], is believed to upregulate cardiovagal tone and has effectively reduced blood pressure in animal studies [4]. Further, VNS has been evaluated as a treatment for heart failure in clinical trials and has demonstrated the ability to significantly increase parasympathetic modulation and improve cardiac structure and function [5]. VNS has exhibited encouraging results for cardiovascular regulation, however, the risks and side effects related to surgical implantation procedures reduce the appeal of this technique [3].

Recently, a non-invasive form of stimulation known as transcutaneous auricular vagus nerve stimulation (taVNS) has been developed as a low-risk alternative to VNS [6]. In taVNS, electrical stimulation is applied to the auricular branch of the vagus nerve (ABVN), which can be accessed through the cymba concha of the ear [7]. Non-invasive, electrical stimulation targeting the ABVN has displayed beneficial effects on the regulation of vagal and sympathetic tone $[8,9]$ and has also been reported to improve cardiac baroreflex sensitivity [10]. These findings provide a promising perspective for the evaluation of taVNS in the regulation of parasympathetic activity as well 
as modulation of blood pressure values in hypertensive patients.

Members of our research team have previously designed a novel taVNS technique that involves gating ABVN stimulation to the exhalatory phase of respiration. This method called Respiratory-gated Auricular Vagal Afferent Nerve Stimulation (RAVANS) has shown to be effective in the activation of brainstem autonomic nuclei such as nucleus tractus solitarii and nucleus ambiguous associated with upregulation of cardiovagal activity $[9,11]$.

In the present study, we aim to identify potential frequency-dependent acute effects of RAVANS on blood pressure regulation in hypertensive patients and define the optimal stimulation protocol for the evaluation of its therapeutic potential in future longitudinal studies.

\section{Materials and methods}

\subsection{Study population}

Twelve subjects $(52.5 \pm 6.0$ years, 8 females $)$ with a diagnosis of primary hypertension were enrolled in the study. All subjects had been prescribed antihypertensive medications and were on stable doses for at least 30 days prior to enrollment. Exclusion criteria consisted of history of other cardio-, cerebro-, or peripheral vascular diseases, diabetes mellitus, morbid obesity $\left(\mathrm{BMI} \geq 40 \mathrm{~kg} / \mathrm{m}^{2}\right)$, secondary hypertension, kidney or liver failure, history of unexplained fainting spells, psychiatric disorders involving psychosis, pregnancy, conditions affecting speech, movement, or the ability to read or follow written instructions, substance use within the previous month, treatment for substance abuse within the previous year, and any metallic implants contraindicating taVNS. Prior to beginning experimental procedures, written informed consent was obtained from research subjects. All study procedures were approved by the local Institutional Review Board.

\subsection{Experimental protocol}

Subjects attended five stimulation sessions in which they received RAVANS at 2, 10, 25, $100 \mathrm{~Hz}$ or Sham stimulation during a given visit. The order of stimulation frequencies was randomized across subjects, and a minimum of 24-hours was set between stimulation sessions to account for potential carry over effects. Custom-built, ergonomically-shaped electrodes (Bionik Medical Devices, Bucaramanga, Colombia) were placed in subjects' left cymba concha (Figure 1). Monophasic rectangular pulses $(300 \mu$ s pulse width, 1s duration) were delivered using a Urostim device (Scwha medico, Germany) with a brief 0.8 s delay after peak inhalation (i.e., during exhalation). Respiratory gating for stimulation was implemented by measuring respiration through a pneumatic belt placed around the subject's lower torax. The respiration signal was acquired by a laptop-controlled device (National Instruments USB DAQCard 6009, Austin, Tx, USA) and computer code (Labview 7.0, National Instruments, Austin, TX, USA) was used to identify end-inhalation in real-time and deliver a TTL signal to a miniature high-frequency relay (Omron Electronics Components, Shaumburg, IL, USA) thereby controlling the electronic gate to pass electrical stimulation to the ear during the exhalatory phase of the respiratory cycle. The intensity of stimulation was adjusted to achieve a moderate, non-painful sensation as indicated by a subjective rating scale. Subjects were asked to inform study staff when the stimulation felt as if it were a ' $5 / 10$ ' on a scale in which ' 0 ' represented no sensation and ' 10 ' represented a sensation that began to feel painful. For Sham stimulation, subjects' sensory thresholds were first identified, then subjects were told that they may or may not feel pulsing in the ear and that we wanted to ensure that the stimulus was not painful. For this session, the electrodes in the ear remained in place, but the leads were disconnected from the stimulator.

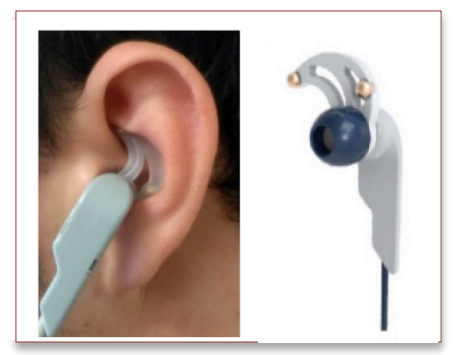

Figure 1. Custom-built electrodes and placement in left ear

The experimental protocol for each session consisted of an initial 10-minute period during which subjects were asked to relax in a seated position, followed by a stimulation period that lasted 30 minutes and a final 10minute recovery period. Arterial blood pressure was recorded continuously throughout sessions using an inflatable finger cuff in conjunction with a Finometer device (Finapress Medical System, the Netherlands). The signal was collected at $400 \mathrm{~Hz}$ using a 16-channel Powerlab DAQ System on a laptop equipped with LabChart Data Acquisition Software (ADInstruments, Colorado, Springs, CO, USA).

\subsection{Statistical analysis}

Continuous blood pressure signals were semiautomatically annotated - systolic peaks were automatically detected and then manually inspected and corrected if needed- with the LabChart Pro Blood Pressure module (ADInstruments, CO, USA). Systolic (SBP), diastolic (DBP), and mean arterial blood pressure (MAP) values were extracted from the annotated data and used for 
statistical testing using STATA (StataCorp, College Station, TX). An analysis was implemented to evaluate significant differences in blood pressure variations for each one of the frequencies evaluated. To this end, mean differences for SBP, DBP and MAP during stimulation (stimulation-baseline) and recovery (recovery-baseline) periods were computed and included in general linear models comparing each of the RAVANS frequencies to the sham session, using Holm's method to correct for multiple testing. In addition, baseline blood pressure values were included in the models and adjusted regression coefficients were calculated. Significance was set as $\mathrm{p}<0.05$.

\section{Results}

Our analysis revealed a significant effect of RAVANS at $100 \mathrm{~Hz}$ in the modulation of SBP values during the stimulation period in comparison with Sham $(\beta=-8.01$, $\mathrm{t}(21)=-2.34, \mathrm{p}=0.029$, Adj $\mathrm{R}^{2}=0.26$ ). Subjects presented an average reduction of $-5.06 \pm 10.55 \mathrm{mmHg}$ during the 100 $\mathrm{Hz}$ session compared with an increase of $2.71 \pm 7.53 \mathrm{mmHg}$ during sham stimulation (Figure 2a).
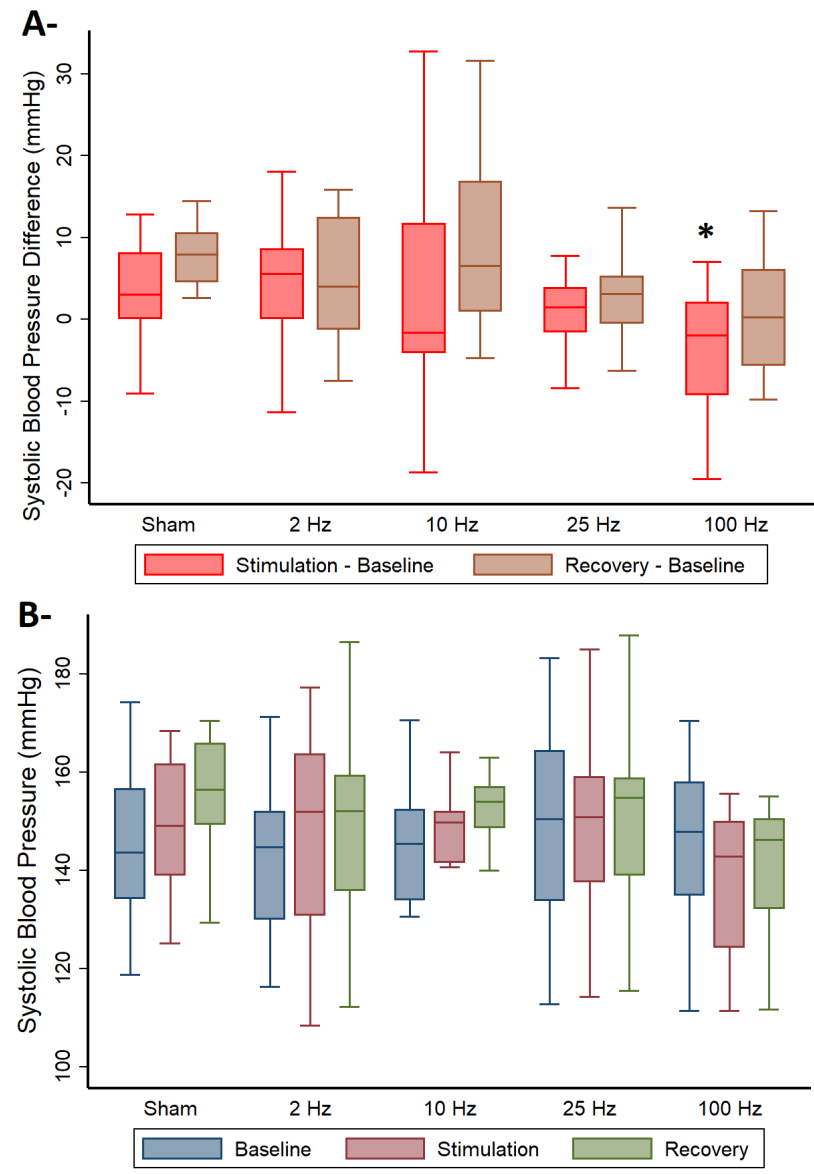

Figure 2. Systolic blood pressure difference (A) and absolute values (B) during experimental sessions with Sham and RAVANS stimulation at frequencies $2,10,25,100 \mathrm{~Hz}$. ${ }^{*} \mathrm{p}<0.05$ when compared to sham stimulation.
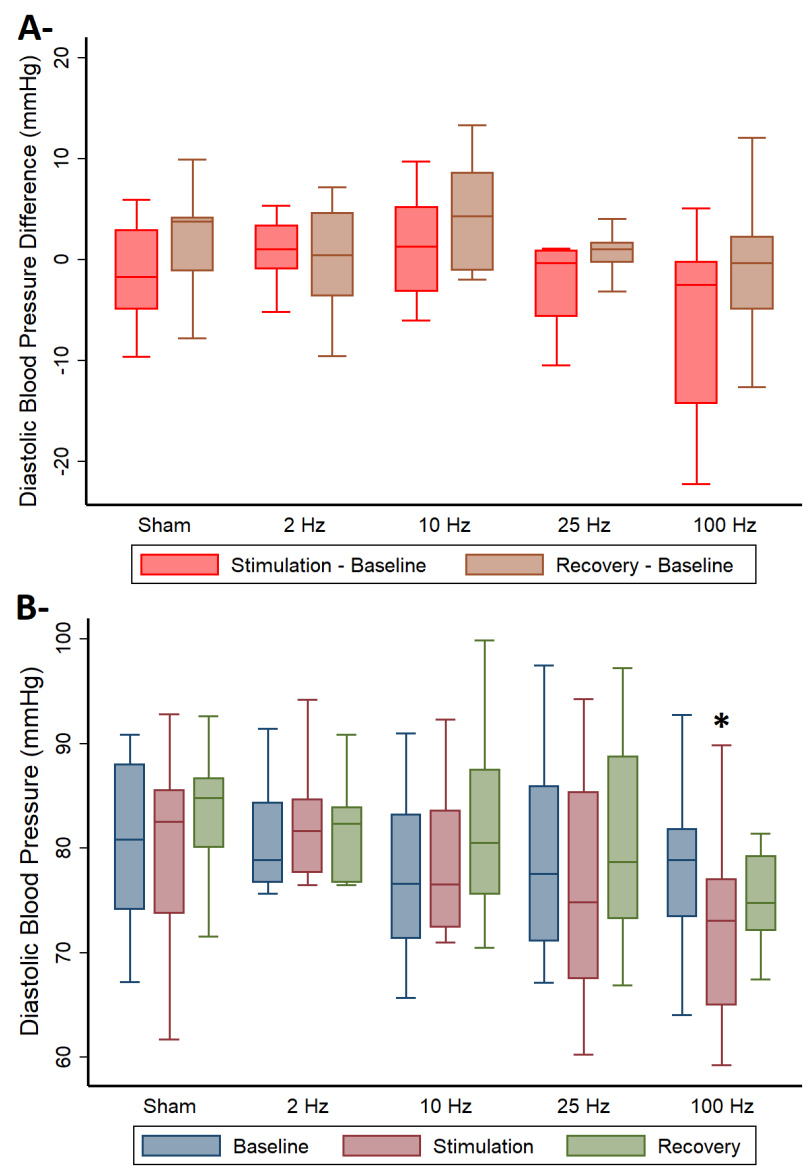

Figure 3. Diastolic blood pressure difference (A) and absolute values (B) during experimental sessions with Sham and RAVANS stimulation at frequencies $2,10,25,100 \mathrm{~Hz} .{ }^{*} \mathrm{p}<0.05$ when compared to baseline values.

There was a trend toward statistical significance for the effects of RAVANS at $100 \mathrm{~Hz}$ on DBP $(\beta=-5.01, \mathrm{t}(21)=-$ 1.84, $\mathrm{p}=0.08$, Adj $\left.\mathrm{R}^{2}=0.23\right)$ and MAP $(\beta=-5.57, \mathrm{t}(21)=-$ 1.97, $\mathrm{p}=0.06$, Adj $\mathrm{R}^{2}=0.28$ ) changes during stimulation in comparison to Sham. In addition, significant lower DBP $(72.4 \pm 9.1$ vs $78.6 \pm 7.9 \mathrm{mmHg}, \mathrm{p}=0.04)$ and $\mathrm{MAP}(94.1 \pm 9.6$ vs $100.3 \pm 9.0 \mathrm{mmHg}, \mathrm{p}=0.04$ ) values were observed during stimulation compared to the baseline period within the 100 $\mathrm{Hz}$ session (Figure 3b). No significant results were observed with other stimulation frequencies.

\section{Discussion}

Our results indicate that respiratory-gated taVNS at 100 $\mathrm{Hz}$ can effectively modulate blood pressure levels in hypertensive patients. No significant effects were found with other stimulation frequencies, suggesting RAVANS at $100 \mathrm{~Hz}$ as the optimal "dose" for further evaluation in hypertension studies. These findings are consistent with a recent neuroimaging study from our group that showed frequency-specific effects of RAVANS on the modulation 
of brainstem autonomic nuclei of healthy subjects [12]. In that report, RAVANS at $100 \mathrm{~Hz}$ was specifically associated with greater activation of the nucleus tractus solitarii and nucleus ambiguous, key components of the vagal brainstem circuitry, when compared to sham or lower stimulation frequencies. These results would support the concept that respiratory-gated taVNS at $100 \mathrm{~Hz}$ could effectively enhance activation of the dorsal vagal complex of the medulla resulting in upregulation of vagal efferent output and modulation of blood pressure levels. However, additional experimental data in animals and humans is required to confirm this hypothesis.

The observed optimized effects of RAVANS at $100 \mathrm{~Hz}$ could be explained by specific targeting of fast adapting, low-threshold mechanoreceptors of the auricular vagus near the dermal/epidermal junction [13]. Receptors called Meissner corpuscles detect "cutaneous flutter" stimuli (5$50 \mathrm{~Hz}$ ), while Pacinian corpuscles lie deeper within the dermis and respond to higher frequencies $(50-400 \mathrm{~Hz})$ of stimulation that are sensed as "vibration." Based on this physiology, the administration of electrical stimulation at a higher frequency range $(>50 \mathrm{~Hz})$ would be ideal for the modulation of $\mathrm{ABVN}$ receptors. In addition, brainstem neurons receiving stimuli transmitted from auricular receptors may also respond in a frequency dependent manner. For instance, studies have found that N-methyl-Daspartate (NMDA) receptors in the nucleus tractus solitarii, activated by glutamate, contribute substantially to neurotransmission at frequencies $>5 \mathrm{~Hz}$, but not at lower frequencies [14]. Such frequency-dependent physiology suggests that modulation of brainstem autonomic nuclei and their regulatory actions on cardiovascular function, including blood pressure modulation, may indeed be optimized with higher stimulation frequencies.

\section{Conclusion}

Our study supports the relevance of stimulation frequency as a key variable for optimization of taVNS effects on blood pressure regulation. Further longitudinal clinical studies will be required to determine the therapeutic value of RAVANS administration at $100 \mathrm{~Hz}$ in hypertensive patients.

\section{Acknowledgments}

This study was supported by the Department of Anesthesia, Critical Care and Pain Medicine at Massachusetts General Hospital; the American Heart Association (Grant No. 16GRNT26420084), NIH-Office of Director (OT2-OD023867), and by the Boston Biomedical Innovation Center (B-BIC), a National Center for Accelerated Innovation funded by the National Heart, Lung, and Blood Institute of the National Institutes of Health under Award Number U54HL119145.

\section{References}

[1] Centers for Disease Control and Prevention NCfHS. Underlying Cause of Death 1999-2003 on CDC Wonder. Online Database, released 2015. 2015 [Available from: http://wonder.cdc.gov/ucd-icd10.html.

[2] Mancia G, Grassi G. The autonomic nervous system and hypertension. Circ Res. 2014;114(11):1804-14.

[3] Daban C, Martinez-Aran A, Cruz N, Vieta E. Safety and efficacy of Vagus Nerve Stimulation in treatment-resistant depression. A systematic review. Journal of affective disorders. 2008;110(1-2):1-15.

[4] Plachta DT, Gierthmuehlen M, Cota O, Espinosa N, Boeser F, Herrera TC, et al. Blood pressure control with selective vagal nerve stimulation and minimal side effects. Journal of neural engineering. 2014;11(3):036011.

[5] Premchand RK, Sharma K, Mittal S, Monteiro R, Dixit S, Libbus I, et al. Autonomic regulation therapy via left or right cervical vagus nerve stimulation in patients with chronic heart failure: results of the ANTHEM-HF trial. J Card Fail. 2014;20(11):808-16.

[6] Ventureyra EC. Transcutaneous vagus nerve stimulation for partial onset seizure therapy. A new concept. Childs Nerv Syst. 2000;16(2):101-2.

[7] Peuker ET, Filler TJ. The nerve supply of the human auricle. Clin Anat. 2002;15(1):35-7.

[8] Clancy JA, Mary DA, Witte KK, Greenwood JP, Deuchars SA, Deuchars J. Non-invasive vagus nerve stimulation in healthy humans reduces sympathetic nerve activity. Brain Stimul. 2014;7(6):871-7.

[9] Sclocco R, Garcia RG, Kettner NW, Isenburg K, Fisher HP, Hubbard CS, et al. The influence of respiration on brainstem and cardiovagal response to auricular vagus nerve stimulation: A multimodal ultrahigh-field (7T) fMRI study. Brain Stimul. 2019;12(4):911-21.

[10] Antonino D, Teixeira AL, Maia-Lopes PM, Souza MC, Sabino-Carvalho JL, Murray AR, et al. Non-invasive vagus nerve stimulation acutely improves spontaneous cardiac baroreflex sensitivity in healthy young men: A randomized placebo-controlled trial. Brain Stimul. 2017;10(5):875-81.

[11] Garcia RG, Lin RL, Lee J, Kim J, Barbieri R, Sclocco R, et al. Modulation of brainstem activity and connectivity by respiratory-gated auricular vagal afferent nerve stimulation in migraine patients. Pain. 2017;158(8):1461-72.

[12] Sclocco R, Garcia RG, Kettner N, Fisher H, Stowell J, Isenburg $\mathrm{K}$, et al. Stimulation frequency affects brain response to respiratory-gated auricular vagal nerve stimulation. 25th OHBM Annual Meeting; 2019.

[13] McGlone F, Reilly D. The cutaneous sensory system. Neuroscience and biobehavioral reviews. 2010;34(2):148 59.

[14] Zhao H, Peters JH, Zhu M, Page SJ, Ritter RC, Appleyard SM. Frequency-dependent facilitation of synaptic throughput via postsynaptic NMDA receptors in the nucleus of the solitary tract. J Physiol. 2015;593(1):111-25.

Address for correspondence:

Riccardo Barbieri

Politecnico di Milano, Via Ponzio 34/5, 20133, Milano, Italy riccardo.barbieri@polimi.it 